\title{
IOT Based Agriculture - A Review
}

\author{
P. Sujatha ${ }^{1 *}$ and S. Sheeba ${ }^{2}$ \\ ${ }^{1}$ (Maths), HC\&RI(W),TNAU,Trichy, India \\ ${ }^{2}(S S \& A C), H C \& R I(W), T N A U, T r i c h y$, India \\ *Corresponding author
}

\section{A B S T R A C T}

\section{Keywords}

Internet of Things

(IoT), Smart

Agriculture, Sensor,

Big Data, Cloud

\section{Article Info}

Accepted:

08 June 2020

Available Online:

10 July 2020
The advancements in technology led to the Internet of things, which is an extension of the network of computers to the network of smart devices. The main objective of these various ICT applications, from a development perspective, is that of Empowering People through Knowledge It provides a comprehensive review of its framework, considerations and implications in implementation. Development of agriculture using technology will be very much useful in cultivation. For a new agricultural area, without knowing or monitoring the important parameters of the soil, cultivation will be difficult and so the farmers suffer financial losses. Another way of expressing it is that of developing in people and in communities a learning and innovation capacity, that increases the effectiveness of their efforts to solve problems and to improve their lives. The paper intends to concise the reader about the IoT technology and its operational requirements in agricultural practices. The working of Agriculture IoT is discussed along with some illustrations.

\section{Introduction}

Agriculture IoT can be seen as a network of sensors, cameras and devices which will work towards a common goal of helping a farmer do his job in an intelligent manner.

These devices will be self-sufficient in a way that they will not need human interference to communicate with each other. In other words, the devices are equipped with the intelligence of knowing the time and reasons for interacting with other devices in the system. As we near the threshold of the 21st century the world is faced with an increasingly complex challenge of feeding its growing population, while assuring an equitable and sustainable development.

Scientific and technological progress is generating the knowledge and the tools to make this possible. 


\section{Elements of IOT}

The whole process revolves around the collection of data for use by the farmers and other stakeholders. This is the most crucial part of its working. The devices used range from sensors to cameras and satellite images. The second part consists of the network which will help transfer the data generated by the devices as mentioned earlier. Different types of network technologies like GSM, LTE, $\mathrm{WiFi}$, 3G etc. may be used depending upon the availability and requirements. The third part consists of data collection and computing technology like the Cloud services.

The cloud servers can be made available independent of the locations and hence most suitable for IoT type of systems. The data can be stored and computed upon on such servers. The cloud services can be taken on a pay-peruse policy as they are becoming popular for this reason. The last part of the system will be the Big Data analytics tools which can work on the vast amount of data generated and stored on the cloud servers, to excavate important patters and trends in the data. For example, weather predictions and market analysis can be done using such tools [1].

\section{IOT innovations in agriculture}

\section{Crop data management}

Current advances in data management are making Smart Farming grow exponentially as data have become the key element in modern agriculture to help producers with critical decision-making. These kind of data-based managed farms rely on data that can increase efficiency by avoiding the misuse of resources and the pollution of the environment. This new philosophy centered on agricultural data has been expressed with several names: Agriculture 4.0, Digital Farming, or Smart Farming, and was born when telematics and data management were combined to the already known concept of Precision Agriculture, improving the accuracy of operations . As a result, Agriculture 4.0 is based on Precision Agriculture principles with producers using systems that generate data in their farms, which will be processed in such a way to make proper strategical and operational decisions.

\section{Sensors}

Sensors are the universal devices to monitor crops and to obtain objective information from them. They are usually integrated in a platform, which is the general term used to name the structures where sensors are placed and carried. These platforms may be attached to off-road vehicles or fixed to the ground within fields such as local weather stations. Various soil sensors are used to measure temperature, moisture and light, humidity and ph value.

The wireless sensor networks have become a more emerging technology in precision agriculture. All sensor nodes deployed in the field, continuously sense soil temperature, soil moisture and air humidity of the agricultural field and transmit this information to base station only when the user defined periodic timer or sensed attributes values exceed desired threshold.

Moisture sensor measures soil moisture content down to $1 \mathrm{~m}$. It serves dual purpose, it can be installed and portable. Measurement of $\mathrm{N}$ (nitrogen), $\mathrm{P}$ (phosphorus) and $\mathrm{K}$ (potassium) contents of soil is necessary to decide how much extra contents of these nutrients are to be added in the soil to increase crop fertility. This improves the quality of the soil which in turn yields a good quality crop. It is based on the colorimetric principle where absorption of light by a solution results in variation in the output of the sensor. 
It helps in determining the $\mathrm{N}, \mathrm{P}, \mathrm{K}$ amounts as high, medium, low, or none. The sensor probe along with proper signal conditioning circuits is built to detect the deficient component of the soil. The system thus designed is advantageous as it reduces the undesired use of fertilizers to be added in the soil. One can properly select the fertilizer quantity to be used for reducing the deficiency in the soil at a particular field.

Soil $\mathrm{pH}$ refers to the acidity or alkalinity of the soil. It is a measure of the concentration of free hydrogen ions $(\mathrm{H}+)$ that are in the soil. Chemistry laboratories generally measure soil $\mathrm{pH}$ using $\mathrm{pH}$ meter with water and calcium chloride. The simplest method is to measure $\mathrm{pHw}$ with a portable $\mathrm{pH}$ meter.

\section{Networking}

Access to information through Agricultural Information Systems (AIS) of different types is the most widely spread service based on ICT applications. These information systems can cover both scientific and technological information (generated by research), as well as socio-economic data, market information and environment management information.

There are different types of information systems, that, in terms of their scope, basically fall into one of three categories: (1) local, corporate (institutional) and/or community-level information systems or services; (2) National/Regional Agricultural Information Systems (NAIS/RAIS); and (3) international databases and/or global networks with information facilities.

With the rapid expansion of the Internet and of web publishing, any of the above can become web-based information systems that provide on-line access to the specific document that is requested, if the document is available on the web.
The web-based information systems are leading to the concept of virtual metadatabases that function as a gateway to the information resources that are located in a given region.

\section{Big data}

Data mining is the computing process of discovering patterns in large data sets involving methods at the intersection of artificial intelligence, machine learning statistics and database system. In agriculture, big data is often viewed as a combination of technology and analytics that can collect and compile novel data and process it in a more useful and timely way to assist decision making.

The big data practice comprises capturing relevant data from a huge number of sources, collecting it today and translating it into actionable information to improve business processes and solve problems at scale and speed.

\section{Cloud computing}

Cloud Computing is emerging today as a commercial infrastructure that eliminates the need for maintaining expensive computing hardware, software, Information technology, staff, infrastructure, recourses and their maintenance. Cloud computing consists of a centralized location to store all the relevant data.

It can include various, Separate databases and Soil-related, weather-related, Research, Crop and Farmers-related data can all be stored at a single location, and data availability can be achieved. This data can be accessed by the end-users such as farmers, experts, consultants, researchers etc easily any time from any location through the devices that are connected to the cloud system. 


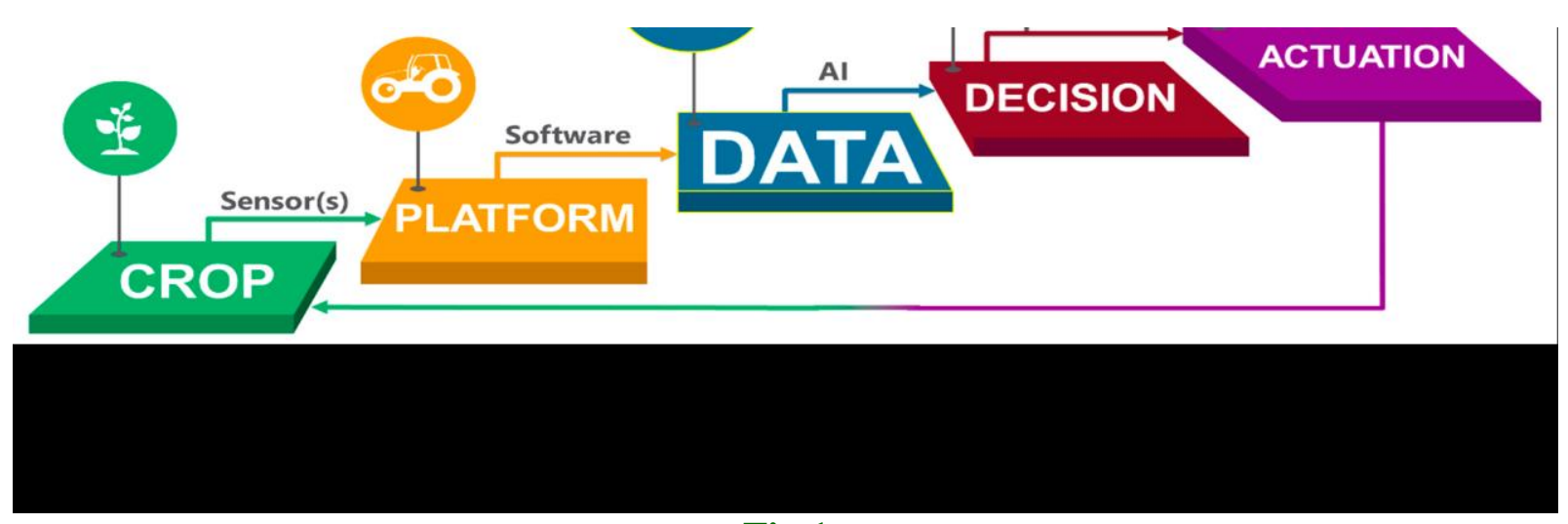

Fig.1

Fig.2 Moisture Sensor

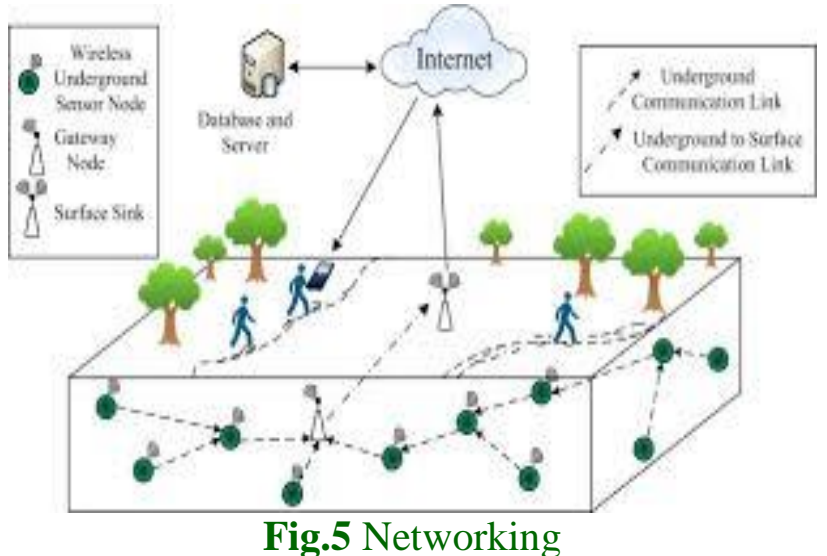




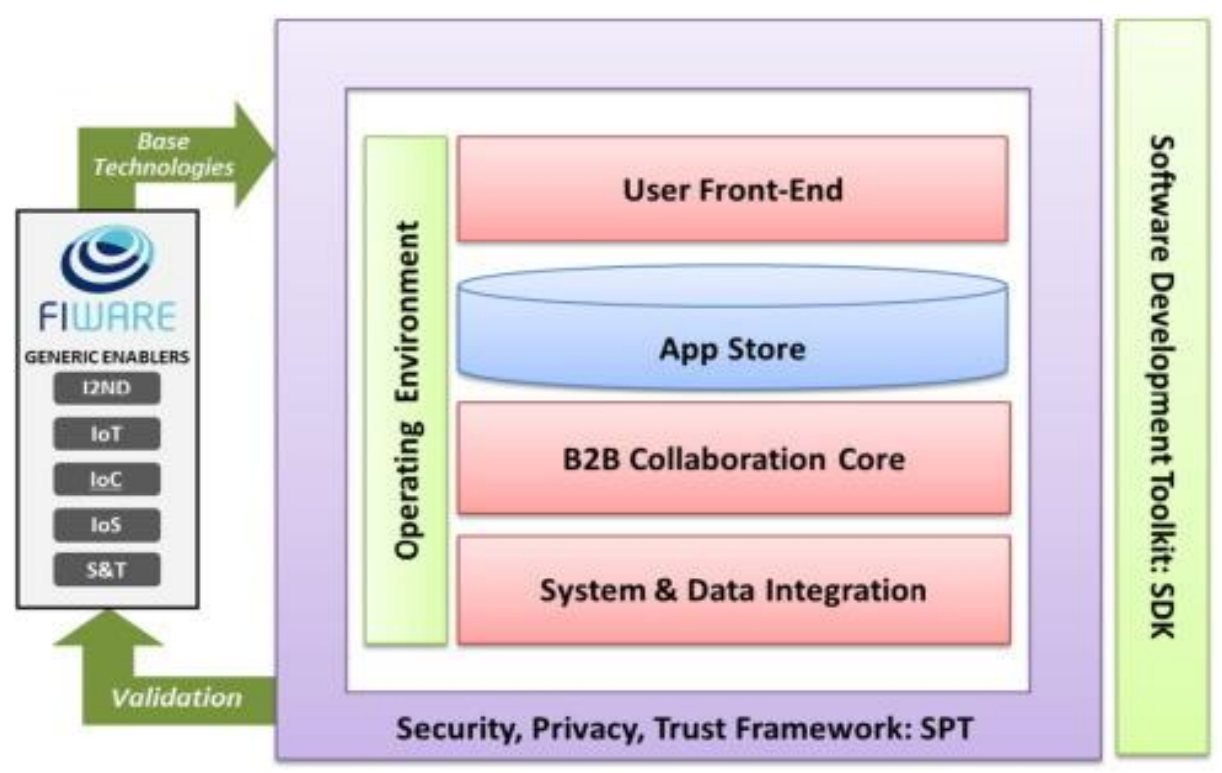

Fig.6 Big Data

Various soil sensors are used to measure temperature, moisture and light, humidity and ph value. The information from the sensors in the soil is sent to the MCP3204 A/D converter then from $A / D$ converter it send to the cloud through Raspberry pi. Finally we can see the information saved to cloud on mobile phone as well as laptop. On the basis of information we know which crop is suitable with given soil parameter. Thus this advanced technology helps the farmers to know the accurate parameters of the soil thus making the soil testing procedure easier.

Agriculture is a field which has relied on traditional methods and experiences till now. But the changing times have exhibited their impact on agricultural practices and they have started to adapt to the dynamics of change. The use of Internet of Things (IoT) in agriculture will not only improve the yields but also effectively manage all the farm activities.

The implementation may have certain implications like cost factor and lack of knowledge but it can nevertheless be worked upon to reap the benefits to the most. There is a dire need to bring about changes in agriculture as a majority of population depends on it for their living. While the vast majority of farmers do large work for maintaining and increasing soil health using conservation practices alone, measurement tools will be instrumental in ensuring a sustainable farming future.

\section{References}

Al-Fuqaha, A., Guizani, M., Mohammadi, M., Aledhari, M. and Ayyash, M. (2015) "Internet of Things: A Surveyon Enabling Technologies, Protocols, and Applications", IEEE Communications Surveys \& Tutorials, Vol. 17 No. 4,pp. 2347-2376. ISSN 1553-877x.FarmBot (2016) "FarmBot genesis", FarmBot [Online].

Lee, Chih-Kung, Julie Lee, Po-Wen Lo, Hsiao-Lin Tang, Wen-Hsin Hsiao, JuiYao Liu, and Ting-Li Lin. "Taiwan Perspective: Developing Smart Living Technology." AUSMT International Journal of Automation and Smart Technology 1.1 (2011): 93-106. Web.

Stočes, Michal, Jiří Vaněk, Jan Masner, and J. Pavlík. "Internet of Things (IoT) in Agriculture - Selected Aspects." Agris 
On-line Papers in Economics and Informatics AOL VIII.1 (2016): 83-88. Web.

Vermesan, O. and Friess, P. (2013) "Internet of things: converging technologies for smart environments and integrated ecosystems", Aalborg Denmark: River Publishers. ISBN 978-87-92982-96-4.
Wu, Ziyu, Shijun Li, Manhua Yu, and Juxin Wu. "The Actuality of Agriculture Internet of Things for Applying and Popularizing in China." Proceedings of the 2015 International Conference on Advances in Mechanical Engineering and Industrial Informatics (2015). Web.

\section{How to cite this article:}

Sujatha. P. and Sheeba. S. 2020. IOT Based Agriculture - A Review. Int.J.Curr.Microbiol.App.Sci. 9(07): 708-713. doi: https://doi.org/10.20546/ijcmas.2020.907.081 